\title{
3D Nanocrystal Images at Atomic Resolution from Diffraction Data using Iterative Algorithms.
}

\author{
J.S. Wu and J.C.H. Spence \\ Department of Physics and Astronomy, \\ Arizona State University, Tempe, AZ 85281-1504, USA
}

The "oversampling" of diffraction data from non-periodic objects allows phase information to be recovered [1] using the iterative Gerchberg-Saxton-Fienup algorithms [2]. These consist of two main parts: error reduction, and hybrid input-output (HIO). The method has been applied to experimental electron and X-ray diffraction data to solve the phase problem and obtain 2D images [3]. Here we use simulations to demonstrate the possibility of reconstructing atomic-resolution images of 3D nano-crystals from their diffraction patterns by diffractive imaging using HIO.

Although the electron exit-face wavefunction $\psi(\boldsymbol{r})$ is in general complex, the "real object" needed by HIO can be obtained in the weak phase object approximation, which we assume. The potential for the crystal was calculated using $\phi(\boldsymbol{r})=\Sigma V_{g} \exp (-2 \pi \mathrm{i} \boldsymbol{r} \cdot \boldsymbol{g})$; each pixel in real space has resolution $0.08 \mathrm{~nm}$. Figure $1(a)$ shows $\phi(r)$ for a nano-crystal of $\mathrm{MgO}$ consisting of $3 \times 3 \times 3$ unit cells. In order to oversample, a larger computational supercell with $6 \times 6 \times 6$ unit cells was used, filled with zeros outside the boundary (support) of the $\mathrm{MgO}$ nano-crystal. The support $S(\boldsymbol{r})$ is defined as the region outside of which the object transmission function is known to be zero. An approximate knowledge of the support (to be obtained from a low resolution TEM image) is needed for HIO. In our simulations, the support is a cube whose size ranges from $3 \times 3 \times 3$ to $4 \times 4 \times 4$ unit cells. The iterations start with the modulus of the Fourier transform of the potential $(F(\boldsymbol{u})=\mathrm{FT}\{\phi(\boldsymbol{r})\})$ ( fig. $1(b)$ ) and random phases. We apply an additional "known sign" constraint to the potential in real space, so that a looser support is permitted. The progress of the iterations can be followed using a normalized root-mean-square $(R M S)$ error, which shows the amount by which the image violates real-space constraints $R M S=\left[\sum_{r \notin S}|\psi(r)|^{2} / \sum_{r}|\psi(r)|^{2}\right]^{1 / 2}$. (RMS includes only the area outside the support). A reciprocal space error metric is also a useful, especially for the error-reduction algorithm: $R_{F}=\left[\sum_{u}|G(u)-F(u)|^{2} / \sum_{u}|F(u)|^{2}\right]^{1 / 2}$. Here $G(\boldsymbol{u})$ is the Fourier transform of current estimate of the exit-wave function. Figure 1(c) shows the reconstructed wave $\psi(\boldsymbol{r})$ after only 200 iterations, and its Fourier transform $G(\boldsymbol{u})$ is shown in fig. $1(d)$. Figure 2 shows the $R M S$ and $R_{F}$ values as a function of iteration number.

In conclusion, atomic resolution tomographic reconstruction of a typical electron microscope sample has been successfully demonstrated from simulated diffraction intensities. This provides the essential tool for the analysis of our experimental data. The preparation of an isolated object is the most challenging aspect of experimental work, which uses the Kohler mode for selected area diffraction from chains of $\mathrm{MgO}$ cubes spanning holes in carbon films. ARO DAAD190010500

\section{REFERENCES}

1. D. Sayre, Acta Cryst., 5 (1952) 843. J. Miao et al. Nature, 400, p. 342 (1999).

2. J. Fienup, Applied Optics, 21 (1982) 2758. Also Spence et al. Phil Trans.360, p. 875 (2002).

3. U.Weierstall et al. Ultramic.90 (2001) 171 Electrons, He et al. Acta Cryst (2003) in press X-rays. 

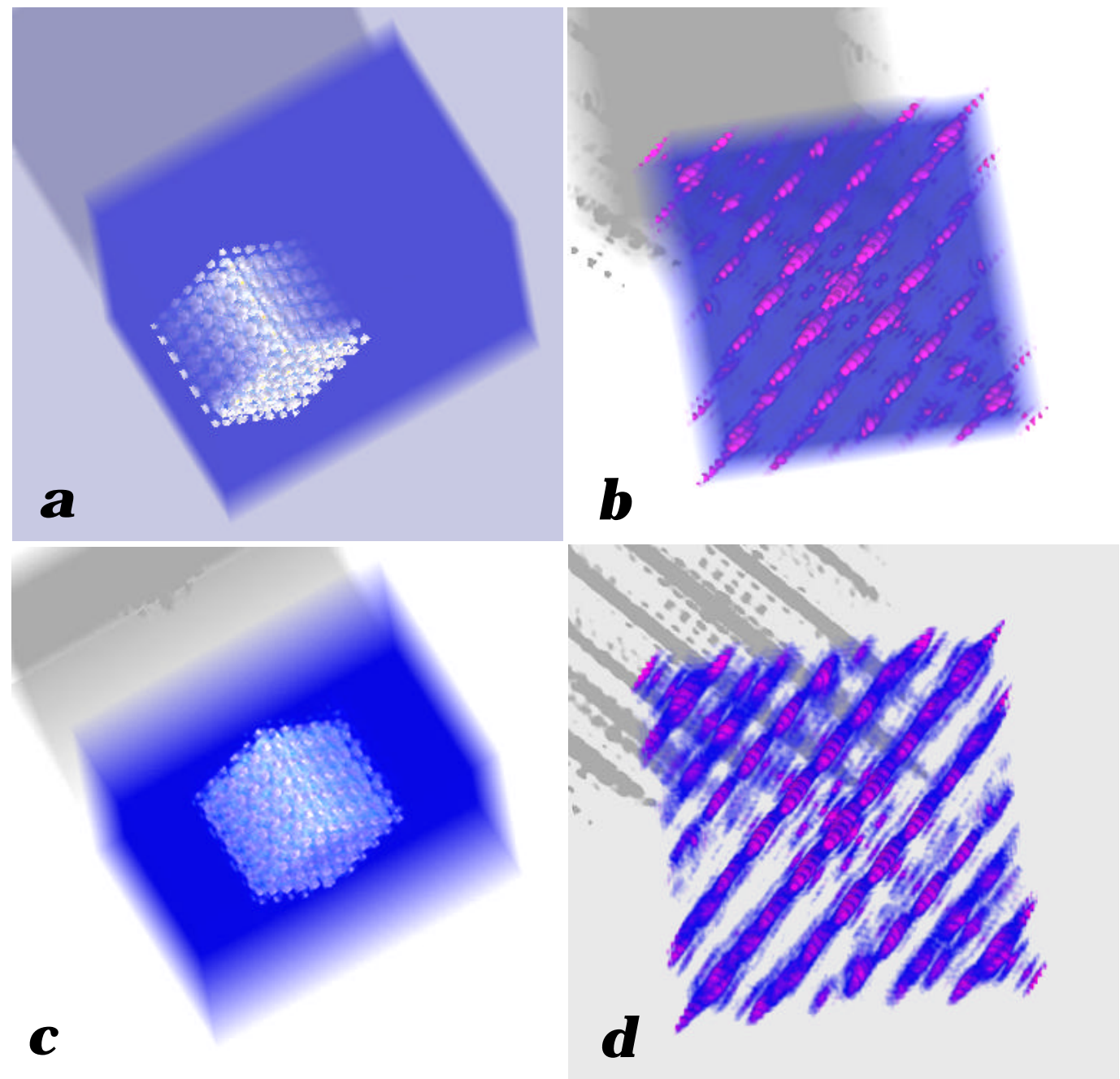

Figure 1. (a) Simulated $\mathrm{MgO}$ nano-crystal with $3 \times 3 \times 3$ unit cells situated at the edge of a cubic of $6 \times 6 \times 6$ unit cells. (b) Modulus of Fourier transform of the cubic in $(a) .(c)$ Reconstructed 3D exit-wave function by 200 times iteration. The support situated in the center of the large cubic. The origin is thus moved compared to $(a)$. (d) Modulus of Fourier transform of the cubic in $(c)$.

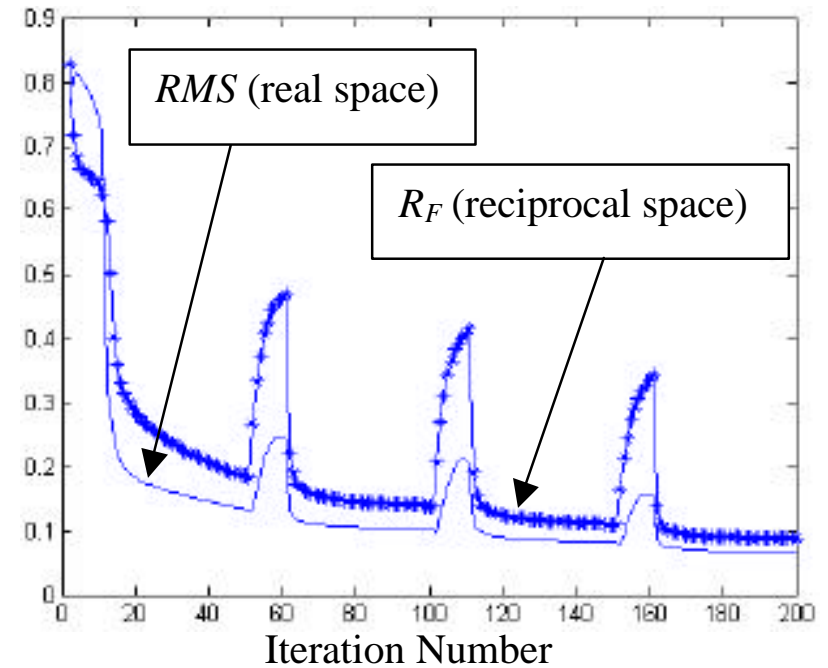

Figure 2. Real space normalized root-mean-square $(R M S)$ and reciprocal space error metric $\left(R_{F}\right)$ as a function of iteration number for reconstruction of fig. 1(c). Although both values were increased when applying the hybrid input-output algorithm comparing to the error-reduction algorithm, it is crucial in finding the global minimum. 